\title{
COMMUNICATION ENTRE POUVOIRS PUBLICS, ENTREPRISES ET ASSOCIATIONS : DU CONFLIT DE LÉGITIMITÉ AU RENFORCEMENT DES LÉGITIMATIONS.
}

\author{
Thierry Libaert et Jean-Marie Pierlot ${ }^{1}$
}

Que se passe-t-il quand des entreprises développent une stratégie de communication au sujet d'un partenariat qu'elles ont entrepris avec une association de protection de l'environnement ou de défense des droits humains ? Quand un gouvernement est accusé par une ONG de ne pas respecter la dignité humaine en enfermant arbitrairement des demandeurs d'asile et envisage une réponse argumentée ?

Quand pouvoirs publics, entreprises et associations entreprennent de réfléchir à leur manière de communiquer à propos des uns et des autres, ils mettent en jeu leur propre légitimité en s'efforçant de l'accroître soit par contagion, soit en se démarquant, voire en s'opposant à des organisations dont les valeurs sont éloignées des leurs. Chacun de ces trois types d'organisation s'efforce de communiquer sur ses activités à partir d'une logique d'action et d'échange différente des deux autres. A travers la coopération ou le conflit, des processus de légitimation renforcent ou affaiblissent les organisations qui en sont les acteurs.

1 Maîtres de conférence invités au Département de Communication, UCL.

Recherches en communication, $\mathrm{n}^{\circ} 25$ (2006). 
Ce texte se propose d'investiguer l'articulation entre logiques d'action et logiques de communication lorsque pouvoirs publics, entreprises et associations décident de se frotter les uns aux autres pour le meilleur et pour le pire. Il examine en particulier deux situations où chacun de ces trois groupes d'acteurs se rencontrent, mettant en œuvre, l'une, une forme de coopération, l'autre, une logique d'affrontement.

\section{Pouvoirs publics, entreprises et associations : des logiques d'échange différentes}

Si l'on se place, à la suite de Karl Polanyi ${ }^{1}$, du point de vue économique pour qualifier le type d'échanges que réalisent pouvoirs publics, entreprises et associations avec leurs différents publics : citoyens, clients, individus ou collectivités, on peut définir la logique des échanges entre l'entreprise et ses publics comme celle de la solvabilité sur la place du marché ; celle des échanges propres à l'Etat et aux pouvoirs publics, par la redistribution des biens et services de la collectivité, sur base de lois et décrets. Pour ce qui concerne les associations, cette logique est généralement plus floue.

Et d'abord, l'univers des associations est habituellement mal défini. On parle spontanément des organisations non-gouvernementales (ONG) ou du secteur non-marchand, comme si ce tiers-secteur ne pouvait être envisagé que par opposition aux deux autres.

Certains auteurs ont tenté de clarifier la terminologie juridique et économique des associations sans but lucratif (asbl) en les situant comme une composante commune du secteur non marchand et de l'économie sociale ${ }^{2}$.

Le sociologue Jean-Louis Laville apporte à la définition des associations une composante plus dynamique d'engagement : "L'association naît d'une absence de lien social vécue comme un manque par des personnes qui s'engagent pour y remédier dans la réalisation d'un bien commun qu'ils déterminent eux-mêmes » 3 .

1 Auteur de La grande Transformation, aux origines politiques et économiques de notre temps, publié en Angleterre dès 1944, mais en version française seulement en 1983 chez Gallimard dans la collection «Bibliothèque des sciences humaines ».

2 Par exemple pour la Belgique, la Fondation Roi Baudouin, Le secteur associatif en Belgique, une analyse quantitative et qualitative, Bruxelles, décembre 2005.

3 Laville, Jean-Louis et Sainsaulieu, Renaud (dir.), Sociologie de l'association, Des organisations à l'épreuve du changement social, Desclée de Brouwer, Paris 1997. 
Si on examine l'ensemble des échanges économiques dans un cadre plus large que celui du seul marché, il est intéressant de se référer à la synthèse qu'a effectuée Karl Polanyi dans «La Grande Transformation $»^{1}$. Il identifie quatre types de comportements économiques, destinés à faire circuler biens et services. Entre ces quatre types, la logique des interactions varie selon les époques :

- Le marché permet une rencontre entre offre et demande de biens et services, échangés à travers la fixation d'un prix.

- La redistribution remet la production entre les mains d'une autorité centrale, chargée de la répartir entre l'ensemble des citoyens, sur base de règles de prélèvements obligatoires (taxes, impôts, ...) et de leurs affectations.

- La réciprocité s'établit sur la volonté prioritaire de manifester un lien entre les parties prenantes. Le principe d'échange est fondé sur le don, appelant un contre-don à la fois libre et obligé ${ }^{2}$, selon la dynamique mise en évidence par Marcel Mauss du donner-recevoir-rendre.

Comme le souligne encore Laville ${ }^{3}$, "Le cycle de la réciprocité s'oppose à l'échange marchand parce qu'il est indissociable des rapports humains qui mettent en jeu des désirs de reconnaissance et de pouvoir et il se distingue de l'échange redistributif parce qu'il n'est pas imposé par un pouvoir central. »

- Enfin, le principe de l'administration domestique renvoie à la production au sein du groupe d'appartenance « naturel » (famille, village ...) qui forme une unité autarcique.

Dans la société moderne, ces principes sont réaménagés selon trois pôles économiques : l'économie marchande, dominée par un marché tout-puissant aspirant à se rendre totalement indépendant des autres fonctions économiques ; l'économie non marchande, en vertu de laquelle la montée de la « question sociale » a obligé l'Etat à contrecarrer les effets destructeurs du libéralisme économique par une redistribution vers les couches sociales les plus fragilisées ${ }^{4}$; et enfin l'économie non moné-

1 Polanyi, Karl, op. Cit.

2 Caillé, Alain, Anthropologie du don, Le tiers paradigme, Desclée de Brouwer, Paris 2000.

3 Jean-Louis Laville et Renaud Sainsaulieu (dir.), op. cit., p. 80.

4 Voir par ex. Donzelot, Jacques, L'invention du social - Essai sur le déclin des passions politiques, Fayard, Paris 1984, ou la revue bimestrielle Politique (Bruxelles) n 37 de décembre 2004 consacrée au $60^{\mathrm{e}}$ anniversaire de la Sécurité sociale en Belgique. 
taire, où la redistribution des biens et services est confiée à la réciprocité et à l'administration domestique.

Cette vision large de l'économie développée par Polanyi, où se retrouvent trois pôles qui ne sont pas étanches les uns aux autres mais peuvent se combiner entre eux, permet de penser une économie plurielle, au lieu de la réduire à la pensée unique du néo-libéralisme.

Mais la dynamique associative ne se cantonne pas dans la seule sphère réciprocitaire. Certaines associations deviennent parfois de quasiadministrations publiques subventionnées ou des quasi-entreprises, quand elles se tournent entièrement vers le marché. Par ailleurs, dans la mobilisation de leurs ressources, les associations combinent bénévolat et autres contributions non monétaires, ressources privées provenant du « fund raising » et du sponsoring/mécénat, ressources publiques liées à la reconnaissance de leurs activités (subsides p. ex.).

Laville : "L'ensemble associatif ne constitue pas pour autant un secteur à part puisqu'il combine la réciprocité initiale avec le marché et la redistribution. ${ }^{1}$.

Ainsi, la proximité entre associations, entreprises et pouvoirs publics peut générer entre elles des relations qui vont du conflit à la coopération. Certains auteurs se sont même plu à relever les contradictions des associations qui ouvrent une main pour recevoir la manne des entreprises et de l'État et ferment le poing pour dénoncer les profits illégitimes des premières et les manquements à la démocratie de l'autre ${ }^{2}$ !

Comment ces trois entités construisent-elles leur communication lors de leurs différentes formes d'échanges, conflictuelles ou coopératrices? Quels risques et opportunités de légitimation pour chacune d'elles à travers leurs actions réciproques?

\section{Echanges entre pouvoirs publics, entreprises et associations et enjeux de légitimation}

\section{Les échanges vus du point de vue des pouvoirs publics}

Des échanges privilégiés entre représentants de l'Etat et entreprises et associations se construisent dans le cadre de partenariats privé/public

1 Laville, J.-L., Idem, p. 84.

2 Pech, Thierry et Padis, Marc-Olivier, Les multinationales du cœur, Les ONG, la politique et le marché, Seuil, Paris 2004. 
(PPP), à tous les niveaux de pouvoir, depuis le niveau le plus centralisé de l'État jusqu'aux communes. Le PPP résulte d'une transformation où l'État, de plénipotentiaire dans la phase où il était reconnu comme ÉtatProvidence ${ }^{1}$, entre de plus en plus dans une logique gestionnaire : de fait, la diminution des ressources propres de l'État l'oblige à s'associer avec des partenaires privés, en adoptant un profil de gestionnaire - qui trouve d'ailleurs son modèle dans l'organisation entrepreneuriale ${ }^{2}$. Ce type de partenariat se multiplie également avec des organisations du secteur associatif, par ex. dans le cadre de projets urbanistiques ou de concertations approfondies avec la population (contrats de rivière, ....). ${ }^{3}$

\section{Les échanges vus du point de vue des entreprises}

Le concept d' " entreprise citoyenne » connaît des effets de mode ${ }^{4}$ au sein des sociétés commerciales et implique une ouverture à l'ensemble de leurs parties prenantes. Ce terme de «parties prenantes » est traduit de l'expression anglaise "stakeholders », elle-même faisant écho au terme «shareholders », actionnaires, l'un des quatre piliers de l'entreprise, aux côtés des fournisseurs, des clients et des salariés. La notion de « partie prenante » s'est progressivement élargie des consommateurs d'abord, aux riverains, aux médias, jusqu'à désigner toute personne ou organisation qui a un intérêt légitime dans un projet ou une entité5.

Les entreprises citoyennes tentent donc d'intégrer un maximum de parties prenantes dans leurs projets et n'hésitent pas à associer riverains, associations de protection de l'environnement et représentants publics à la mise en œuvre de nouveaux marchés ou d'une nouvelle implantation d'usine, par exemple.

1 Rosanvallon, Pierre, La nouvelle question sociale - Repenser l'État-providence, Seuil, Paris 1995

2 Vrancken, Didier, Les métamorphoses de l'administration, Labor, Bruxelles 2005, p. 78.

3 Voir Lascoumes, Pierre, L'éco-pouvoir, Environnements et politiques, La Découverte, Paris 1994.

4 On parle beaucoup aussi de R.S.E., responsabilité sociétale des entreprises, ou d'E.S.R., entreprises socialement responsables.

5 Bonnafous-Boucher, Maria et Pesqueux, Yvon (dir.), Décider avec les parties prenantes - Approches d'une nouvelle théorie de la société civile, La Découverte, Recherches, Paris 2006. 


\section{Les échanges vues du point de vue des associations}

Dans leur préoccupation d'élargir les droits humains à ceux qui ne sont considérés ni comme des clients solvables ni comme des citoyens à part entière payant leurs impôts, les associations tentent d'obtenir des pouvoirs publics et des entreprises une reconnaissance de leur action, soit par un dialogue et un partenariat constructifs, soit par une interpellation faisant appel au comportement citoyen, voire civique, de la part de leurs interlocuteurs publics ou privés.

Ces associations tentent de mobiliser à leur cause un maximum de sympathisants pour rendre visible leur représentativité et acquérir par là une légitimité suffisante pour obtenir gain de cause.

\section{Transferts de légitimité entre les organisations dans leurs échanges}

Lorsqu'ils tentent d'entrer en relation avec des organisations dont la logique des échanges est différente de la leur, les pouvoirs publics, entreprises ou associations courent un risque de réduire leur capital de légitimité acquise.

Ils espèrent cependant accroître leur légitimité en capitalisant leur propre légitimité avec celle du partenaire lorsque l'interaction se déroule dans le registre de la coopération. En revanche, une interaction de type conflictuel risque de renforcer la légitimité d'un des partenaires et de réduire celle de l'autre. L'état actuel de la répartition des légitimités indique une forte inégalité. Pour reprendre l'étude de l'Annual Trust Barometer publiée en 2006 ', les ONG bénéficient en France d'une perception de confiance deux fois supérieure à celle des entreprises. De fait, ce sont ces dernières qui paraissent en position de demandeur. Le partenariat s'effectue alors souvent dans une vision de transfert de légitimité comme si la relation avec l'association pouvait conférer à l'entreprise l'équivalent d'un label réputationnel².

1 The Annual Trust Barometer est une étude réalisée chaque année auprès de 2000 cadres supérieurs et leaders d'opinion des principaux pays industrialisés. La $8^{\text {ème }}$

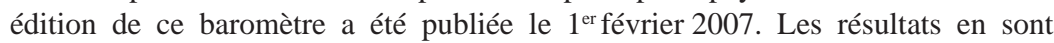
disponibles sur le site www.edelman.com

2 Valérie Swaen a tenté de mesurer par un dispositif expérimental la réaction des consommateurs à l'activité des entreprises qui se présentent à eux comme « socialement responsables » - voir Swaen V. et Vanhamme J. (2003), L'utilisation de 


\section{Coopération}

Lorsqu'une organisation publique s'engage dans un partenariat privépublic, elle s'enquiert de la bonne réputation de son partenaire, sous peine d'être associée à l'image négative que celui-ci peut produire, en cas de difficultés financières par exemple. Lorsqu'une entreprise tente d'associer différentes parties prenantes à son projet, elle s'assure que chacune d'elles adhère au projet, sans quoi l'une d'elles peut convaincre plusieurs autres de se liguer contre le promoteur du partenariat. Lorsqu'une association s'associe à une entreprise pour la promotion de droits humains ou de l'environnement, elle espère ne pas s'engager dans un marché de dupes afin de ne pas ternir sa réputation ${ }^{1}$. Le conflit n'est, on le voit, pas nécessairement éloigné de la coopération. L'étude de Valérie Swaen montre d'ailleurs que les entreprises se présentant elles-mêmes comme socialement responsables encourent un risque bien plus grand d'être désavouées par le public en cas d'écart à ce principe que celles qui ne se déclarent pas comme telles.

\section{Conflit}

Dans le cas d'interactions de type conflictuel, un des partenaires de l'échange tente d'accroître sa légitimité en dénonçant la dérive de l'autre ou des autres partenaires par rapport à ses propres valeurs. Ainsi, une entreprise ou un de ses porte-parole peut reprocher à un Etat de limiter le libre jeu du marché ; une association peut accuser une entreprise de produire des biens ou services au détriment des droits élémentaires de ses travailleurs ; un représentant des pouvoirs publics peut accuser une association de refuser le jeu de la citoyenneté en ne respectant pas les règles de l'Etat de droit.

L'étude des relations ONG/Etat/Entreprises indique que ces deux pôles sont rarement figés mais évoluent dans une relation dynamique oscillant en permanence selon la transformation du contexte médiatique, des attentes de l'opinion publique et du jeu des acteurs internes. La coopération apparaît être un idéal difficile à atteindre. Il y a dans la psychologie sociale propre à de nombreuses associations une propension au combat pour des motifs de cohésion des adhérents et de médiatisation externe ${ }^{2}$.

l'argument « citoyen » dans les campagnes de communication : analyse des risques dans la perspective d'une crise, Working Paper IAG 84/02, Institut d'Administration et de Gestion, Université catholique de Louvain, Louvain-la-Neuve.

1 Entreprises et Mécénat $\mathrm{N}^{\circ} 107$, Paris Juin 2005, page 16.

2 Voir en ce sens Libaert, Thierry. La communication verte, Editions Liaisons, Paris 1992, pp. 69 à 73. 
En outre, le conflit ne doit pas être interprété autour d'une immédiate négativité : «On y voit toujours la trace d'un échec, alors qu'il est un moyen de visualiser le heurt de plusieurs représentations $»^{1}$.

Le conflit peut porter explicitement sur la légitimité de l'un par rapport à l'autre. Certains ministres ont ainsi dénoncé le caractère non représentatif d'associations en vertu du caractère non élu de leurs représentants ${ }^{2}$; des associations reprochent à des entreprises de bafouer l'intérêt général en se contentant de satisfaire leurs seuls actionnaires et en délocalisant l'emploi ; des porte-parole d'entreprises invalident le caractère scientifique des analyses réalisées par des associations en les déclarant non fondées. Les entreprises exigent de plus en plus que les ONG se prêtent à la même transparence que ces dernières leur réclament et tendent à diffuser des procédures de notation des acteurs du milieu associatif à l'exemple des agences de notation sociale 3 . Plus largement, l'idéologie néo-libérale dénie le droit aux Etats de réguler la vie économique 4; l'idéologie de l'Etat social actif conteste la dynamique syndicale; l'idéologie altermondialiste refuse la mondialisation privatisante du marché des biens et services.

\section{Etudes de cas : coopération et/ou conflit}

\section{Café et commerce équitable ${ }^{5}$ : les pouvoirs publics clients des entreprises et des associations}

Depuis quelques années, on voit fleurir au sein d'entreprises commerciales diverses initiatives de production de labels se reven-

1 Wolton, Dominique, La dernière utopie, Flammarion, Paris 1993, p. 22. Dans le même sens : Hunyadi, Mark, La vertu du conflit, Pour une morale de la médiation, Cerf, Paris 1995.

2 Pech, Thierry et Padis, Marc-Olivier, op. cit., chapitre II : Les conflits de légitimité (p. 39 sv.)

3 Voir par ex. le site www.ngowatch.org, financé par les entreprises américaines, et dont l'objectif est "Highlighting issues of transparency and accountability in the operations of non-governemental organizations (NGOs) and international organizations (IOs). »

4 Cfr. l'affaire Enron, qui a fait le succès puis la descente aux enfers de cette entreprise qui a crû sur la dérégulation du marché de l'électricité aux Etats-Unis ou, plus proche, les débats autour du projet de fusion entre Suez et Gaz de France.

5 Cette analyse se base sur le dossier Le Fair Trade sera-t-il victime de son succès?, réalisé par Pierre Biélande pour l'agence Alter, Alter Business News for a Sustainable Economy, $\mathrm{n}^{\circ} 100$, octobre 2005 (voir le site www.alterbusinessnews.be). 
diquant du commerce équitable. Ainsi, le négociant anversois Efico $^{1}$, importateur de café en Belgique et approvisionneur de plus de trois cents usines de torréfaction en Europe, a lancé son label de café Sustainable, Fair \& Free Trade, aussitôt contesté par plusieurs ONG concernées par cette activité. Pourtant, lorsque le parlement belge lance en 2005 un appel d'offre pour la fourniture de café issu du commerce équitable, c'est le partenaire commercial qui emporte le marché et non les distributeurs du café labellisé Max Havelaar.

En cause, une définition du commerce équitable suffisamment floue $^{2}$ pour permettre des interprétations fort divergentes et ouvrir la porte à une surprenante concurrence entre ONG et entreprises commerciales sur ce segment de marché. Si les premières disposent d'une expertise incontestable dans le domaine du plaidoyer (lobbying), elles n'ont évidemment pas l'expérience ni la logistique en matière de marques des entreprises commerciales. ${ }^{3}$

L'extension de produits issus du commerce équitable dans les grandes surfaces de distribution est certes une opportunité pour les ONG de populariser un de leurs thèmes de prédilection; en revanche, le risque est de voir les entreprises bénéficier de l'image liée au commerce équitable sans en faire véritablement. Pire : la confusion créée autour de ce concept peut l'affaiblir et le dissoudre dans l'insignifiance.

Faut-il laisser, sans intervenir, les labels se multiplier? N'est-ce pas le rôle de l'Etat de réguler ces diverses initiatives, par exemple en faisant procéder à l'évaluation des différents procédés de labellisation ${ }^{4}$ ? On peut cependant douter du sérieux avec lequel il va jouer son rôle d'arbitre, lorsqu'on sait que dans un autre secteur, celui de la réglementation de la gestion durable des forêts, les autorités publiques (de la Région wallonne, notamment) ont cautionné un label élaboré «sur mesure » par les principaux producteurs de bois, le PEFC (pour Pan

1 Voir le site www.efico.com.

2 dossier « Le Fair Trade sera-t-il victime ... », op. cit. p. 10.

3 «Les ONG sont très compétentes dans le domaine du lobbying afin d'influencer l'opinion publique et de contribuer au débat politique. C'est leur force. Ceci étant, rares sont les personnes travaillant dans les $O N G$ et se levant le matin en pensant comment elles peuvent augmenter leur part du marché.", propos de Lawrence Watson, associé de l'International Federation for Alternative Trade, rapportés dans le dossier "Le Fair Trade sera-t-il victime ...", p. 21.

4 De nombreuses initiatives parlementaires tentent d'ailleurs de fixer par la loi le terme de « commerce équitable ». 
European Forest Certification). Celui-ci a été créé en réplique au $\mathrm{FSC}^{1}$ (Forest Stewardship Council), un label au cahier des charges bien plus contraignant, créé il y a une quinzaine d'années avec les encouragements du WWF et de Greenpeace.

On le voit, à travers cet exemple sur le commerce équitable du café, trois logiques d'action différentes sont en jeu, correspondant aux enjeux de légitimité des trois types d'organisations en présence: pouvoirs publics, entreprises et associations.

Pour l'Etat, il s'agit d'adhérer à un principe de citoyenneté dont les règles satisfont au cahier des charges consigné dans un appel d'offre. Pour l'entreprise, l'objectif est d'accroître ses parts de marché en élaborant un dispositif qui satisfasse la demande des consommateurs, tout en préservant sa légitimité engageant sa responsabilité sociétale. Pour le secteur associatif, il faut élargir un principe de solidarité (en l'occurrence, avec les producteurs de café au Sud) en y intéressant les entreprises et les pouvoirs publics.

Chacun de ces trois partenaires doit mesurer les risques de ce partenariat : l'Etat risque d'être soupçonné de favoriser le marché des entreprises en écartant celui des associations et donc, de faire le jeu du capitalisme international en prétendant faire celui du commerce équitable ; les entreprises risquent de voir leur labellisation dénoncée comme étant une pâle copie d'un principe de solidarité avec les producteurs de café ; les associations peuvent se voir reprocher de faire trop de concessions à leurs principes en s'associant à des partenaires commerciaux.

\section{Le désamiantage du Clémenceau : chronique d'un conflit annoncé}

En février 2006, le porte-avion Clémenceau quitte les côtes françaises à destination de l'Inde avec son démantèlement pour objectif. Cette épopée avait dès l'origine tous les ingrédients d'une crise médiatique, politique et diplomatique et elle se terminera par un retour sur les côtes françaises trois mois plus tard et sans qu'aucune solution n'ait été trouvée. Les protagonistes l'avaient d'ailleurs anticipée et une agence de relations publiques spécialisée dans la problématique de crise fut ainsi retenue. Au coeur du conflit; l'amiante, symbole parfait d'une opacité communicationnelle des pouvoirs publics et en trame de fond

1 www.pefc.org; www.fsc.org 
les relations Nord-Sud, les problématiques de délocalisation et bien sûr la problématique d'armement. Tout fut en place pour une crise majeure et celle-ci advint. La question est : pouvait-il en être autrement ?

Toute cette affaire reposait sur un péché originel ; celui de la méconnaissance par les pouvoirs publics et les industriels concernés de la quantité d'amiante à bord du navire ${ }^{1}$. Si techniquement, la question peut sembler mineure, en termes symboliques elle traduisait au mieux une légèreté dans la connaissance du dossier, au pire le paradigme d'un nouveau mensonge. La bataille de l'image fut rapidement gagnée et l'on observe à quel point la médiatisation d'un zodiac obstruant le passage d'un porte-avion peut apparaître redoutable.

Car différentes stratégies de communication sont au cœur de cette triangulation relationnelle et la visualisation en boucle, sur toutes les chaînes de télévision, d'images de chantiers indiens de démolition, datant pourtant de plusieurs années, a pu donner la victoire à Greenpeace contre l'armée française. Celle-ci essaya bien de communiquer pour dépasser les représentations d'un continent indien, poubelle à amiante, où les travailleurs seraient exposés à tous les dangers, en diffusant le message d'une Inde prête à développer les programmes nucléaires de très haute technologie en coopération avec les Etats-Unis, une Inde qui dans le même temps lançait une OPA pour conquérir l'acier européen ; mais il était trop tard. L'Etat français tenta ensuite tout aussi vainement d'entreprendre une stratégie de détournement des erreurs en reportant les accusations sur les entreprises de désamiantage qui auraient sous-estimé - volontairement ou non - les quantités d'amiante à bord. Toutefois, celles-ci demeurèrent prudemment silencieuses et le conflit resta alors dans une relation Etat/ONG.

L'ultime tentative fut de jouer sur les conflits de légitimité en faisant s'exprimer d'autres associations écologistes comme Robin des Bois dans un sens favorable au désamiantage indien du porte-avion. Parmi les nombreux enseignements de ce cas, il apparaît que si l'Etat français s'est montré en cohérence forte avec les entreprises de désamiantage, la

1 Selon Le Monde du 16/02/06, l'Etat français n'a cessé d'annoncer des chiffres différents selon les circonstances : lors de l'appel d'offre pour le désamiantage en 2003, il indique 215 tonnes. Quelques mois plus tard, le tonnage d'amiante est estimé à la baisse, environ 160 tonnes. Mais en 2005, nouveau chiffre : l'Etat annonce que 270 tonnes de « déchets amiantés » ont été retirés du bateau. Il resterait alors 22 tonnes d'amiante résiduel. Enfin, en 2006, la ministre de la défense avance le chiffre de 45 tonnes ... soit plus de $0,1 \%$ du poids total du bateau, ce qui le fait considérer comme déchet au regard de la législation européenne - donc non exportable. 
cohésion s'est progressivement fissurée et il semble qu'au sein même de l'Etat français, de nombreux acteurs se sont rapprochés progressivement des thèses de Greenpeace, étonnamment très bien renseignés. La légitimité étant totalement passée dans le camp de l'ONG, l'Etat français ne pouvait plus ensuite que conclure et renoncer à l'envoi en Inde du navire, perçu non plus comme l'un des bateaux les plus prestigieux de l'armée, mais comme un déchet amianté que l'on chercherait honteusement à envoyer le plus loin possible.

\section{Conclusion : l'émergence de nouveaux acteurs dans la dynamique de la communication}

Loin d'une vision schématique où les frontières entre les trois acteurs : Etat-Entreprises-associations, seraient étanches, l'analyse des interactions récentes entre ces pôles indique plutôt une porosité qui s'amplifie. L'Etat se décharge de certaines de ses prérogatives d'intérêt général au profit des associations ${ }^{1}$ et des entreprises et dans le même temps sa communication s'effectue de plus en plus sur le registre de l'efficacité et de la performance, valeurs initiales de la sphère entrepreneuriale. L'association fonctionne de plus en plus sur une logique marchande ${ }^{2}$ et l'entreprise, sous l'égide de valeurs de développement durable, se positionne sur une thématique de transparence et de citoyenneté ${ }^{3}$.

De fait, la visibilité des trois pôles se réduit et consécutivement le curseur de l'axe coopération - conflit devient plus instable. Les trois parties prenantes apparaissent en reconfiguration permanente de leurs stratégies d'alliance/d'affrontement et le positionnement de chacun se modifie alors au gré des sujets de rencontre ; la coopération n'est pas exclusive de conflits sous-jacents. La communication apparait souvent comme le point de basculement : parce qu'il existe un risque réputationnel attaché à la coopération, les liens se distendent et le conflit apparaît. C'est aussi parce que l'entreprise évalue le risque d'une attaque sur son image en raison de son éloignement de la sphère de légitimation

1 Sur l'intervention financière accrue des Etats dans l'activité des ONG, dans les secteurs du développement et de l'aide d'urgence, voir Les multinationales du cœur, déjà cité, p. $20 \mathrm{sv}$.

2 Dacheux, Eric, Associations et communication, Critique du marketing, CNRS Editions, Paris 1998.

3 Richebois, Véronique, Les sujets de société inspirent les entreprises, Les Echos, 9 mars 2006, p. 12 
associative par la logique même de l'échange marchand dans laquelle elle s'inscrit, qu'elle visera à nouer une relation de coopération.

Lorsque les grandes associations internationales sont apparues au début des années '70 (Amnesty, Greenpeace, Médecins sans Frontières,...), la configuration apparaissait bipolaire et opposait plutôt le secteur associatif à la sphère politico-industrielle. L'État se positionnait clairement et ouvertement en appui au monde de l'entreprise. La mondialisation, l'explosion des nouveaux médias ont considérablement modifié cette donne et fait émerger deux nouveaux acteurs.

D'abord, et c'est là le point fondamental, l'élargissement de la sphère médiatique a fait surgir l'opinion publique au centre des relations entre les trois acteurs. Parce que chaque enjeu devient potentiellement un objet de forte médiatisation, l'État a tendance à évaluer au coup par coup ses alliances en pratiquant une démocratie d'opinion, où il mesure les risques de perte de légitimité lorsque sa réputation risque de pâtir d'une alliance à tout prix avec des entreprises ${ }^{1}$. Cet enjeu de réputation s'est en outre désormais totalement installé dans le monde des entreprises, où les affrontements avec les associations actives dans les secteurs des droits de l'homme et de l'environnement ont suscité parmi quelques multinationales (Nike, Monsanto, Total ...) des inquiétudes croissantes. D'une rencontre entre la démocratie d'opinion et d'un capitalisme où l'image de marque constitue plus que jamais une valeur stratégique ${ }^{2}$ est né ce que T. Pech et M.-O. Padis n'hésitent pas à nommer un « capitalisme d'opinion $»^{3}$. Ce mouvement de focalisation sur l'image correspond à l'éclatement du monde industriel classique et l'apparition de grands noms de marque contractualisant en permanence les meilleures conditions de production avec des fournisseurs temporaires. Ce primat de l'image dans ce nouveau paysage industriel que le président d'Alcatel prédisait «fabless » (sans usine en appartenance propre) connaît une extension récente avec les tentatives de quantifier la valeur de cette image d'entreprise. Les valeurs obtenues apparaissent souvent considérables $^{4}$. L'image devenant le seul lien d'un réseau épars d'installations de

1 voir ci-dessus l'analyse de l'Affaire du Clémenceau où, au moment où le risque d'image pour l'Etat devient trop important, il en vient à se soumettre à l'argumentation associative.

2 voir les nombreuses études de J.N. Kapferer sur la stratégie des marques et, dans un registre plus militant, le livre de Klein, Naomi, No Logo, La tyrannie des marques, Ed. Léméac/Actes Sud, Arles 2001.

3 Op. cit. p. 69.

4 L'image de Coca-Cola est ainsi estimée à 67 milliards de \$, celle de Microsoft à 59 milliards, cf Business Week, septembre 2005, p. 54 à 61. 
production et de distribution, on comprend dès lors que la coopération des entreprises avec l'univers des associations semble majoritairement motivée par des considérations de communication.

Un autre acteur est alors appelé en arbitre permanent : l'expert. Chacun des trois partenaires cherche la crédibilité auprès de celui qui apparaîtra extérieur à cette interrelation et, lorsque la situation évolue vers une conflictualité, chacun semble se dissimuler derrière la parole de ses propres experts, dont les évaluations souvent contradictoires interrogent et renouvellent leur positionnement dans le débat démocratique $^{1}$.

Ainsi une nouvelle carte des acteurs se met en place dans laquelle l'opinion publique retrouve un rôle central ${ }^{2}$ en raison de son impact potentiellement critique sur l'image des entreprises et celle de ses dirigeants.

La démocratie sera-t-elle dès lors sauvée par la communication? Les experts les plus renommés du capitalisme d'aujourd'hui ${ }^{3}$ proclament eux-mêmes haut et fort que la société néo-libérale ira droit dans le mur, si elle ne s'inscrit pas dans l'horizon du développement durable. Effectivement, la problématique du développement durable, qui combine préoccupations économiques, sociales et environnementales, est un enjeu essentiel pour la dynamique d'ensemble de la société. Cet enjeu ne pourra être rencontré que si la communication cesse d'être conçue sous la seule vision instrumentale des relations entre organisations, mais comme un enjeu même du développement durable dont elle forme vraisemblablement un des pôles, à l'instar des domaines économiques, sociaux et environnementaux

1 Latour, Bruno, Politiques de la nature, Comment faire entrer les sciences en démocratie, Ed. La Découverte, Paris 1999. Pour une étude de cas consacrée aux O.G.M., voir L'opinion publique face aux plantes transgéniques, Entre incertitudes et prises de conscience, Colloque de La Villette, Albin Michel, Paris 1999.

2 Pech, T. et Padis ; M.-O.: « (...) Il faut reconnaître qu'en mobilisant les opinions publiques, les grandes ONG contribuent à réinscrire le capitalisme dans un débat démocratique en resserrant l'articulation entre l'espace public de la discussion et l'espace privé de l'échange marchand. » p. 70.

3 Par exemple, Stiglitz, Joseph E., Quand le capitalisme perd la tête, Fayard, Paris 2003, ou Peyrelevade, Jean, Le capitalisme total, Seuil, Paris 2005. 